\title{
Bounds of the Coefficient Estimates for a Subclass of Bi-Univalent Functions
}

\author{
Nurdiana Nurali ${ }^{1}{ }^{*}$, Aini Janteng ${ }^{2}$ and Rashidah Omar ${ }^{3}$ \\ ${ }^{1}$ Faculty of Science and Natural Resources, Universiti Malaysia Sabah, 88400 Kota Kinabalu, \\ Sabah, Malaysia \\ ${ }^{2}$ Faculty of Science and Natural Resources, Universiti Malaysia Sabah, 88400 Kota Kinabalu, \\ Sabah, Malaysia \\ ${ }^{3}$ Faculty of Computer and Mathematical Sciences, Universiti Teknologi MARA Cawangan Sabah, \\ 88997 Kota Kinabalu, Sabah, Malaysia
}

\begin{abstract}
In this paper, subclass of the class $\varsigma$ of bi-univalent function is introduced using subordination. For functions in the class $\varsigma$, theestimates on the Taylor-Maclaurin coefficients and upper bound of the Fekete-Szegö functional are obtained.
\end{abstract}

Keywords: analytic, bi-univalent, subordination, Fekete-Szegö

\section{INTRODUCTION}

Let $\mathrm{A}$ denote the class of functions in the form

$$
f(z)=z+\sum_{n=2}^{\infty} a_{n} z^{n}
$$

which are analytic and normalized by $f(0)=0$ and $f^{\prime}(0)=$ 1 in the open unit disk $\Delta:=\{z: z \in C$ and $|z|<1\}$. Let $S$ denote the subclass of A. Functions in Sare univalent.

Let $S_{\mathcal{S}}^{*}$ be the subclass belonging to $S$ in the form (1) satisfying

$$
\operatorname{Re}\left(\frac{z f^{\prime}(z)}{f(z)-f(-z)}\right)>0
$$

for $z \in \Delta$. Sakaguchi (1959) introduced functions of starlike with respect to symmetric points as stated in (2). Das \& Singh (1977) then introduced another class $C_{\mathcal{S}}$ namely, convex functions with respect to symmetric points and satisfying the condition

$$
\operatorname{Re}\left(\frac{\left(z f^{\prime}(z)\right)^{\prime}}{(f(z)-f(-z))^{\prime}}\right)>0
$$

for $z \in \Delta$.
If $f \in S$, then there exists an inverse function, $f^{-1}$ which is also univalent in $\Delta$.A function $f \in \mathrm{A}$ is called biunivalent in $\Delta$ if both $f$ and $f^{-1}$ are univalent in $\Delta$. Thus, $\varsigma$ denote the class of bi-univalent functions defined in $\Delta$. All bi-univalent functions have an inverse function with the Taylor series in the form

$$
f^{-1}(w)=w-a_{2} w^{2}+\left(2 a_{2}^{2}-a_{3}\right) w^{3}-\cdots
$$

Many earlier researches published papers concerning bi-univalent functions. These researchers focused on problems connected with coefficients. This research was initiated by Lewin in 1967. Brannan \& Taha (1986) instigated certain subclasses of bi-univalent functions including of starlike, strongly starlike and convex functions which have the similarities with subclasses of univalent functions.

A function $f$ is said to be subordinate to function $g$ if both functions are analytic in $\Delta$, and if there is exists function $w$, defined on $\Delta$ with $w(0)=0$ and $|w(z)|<1$ satisfying $f(z)=g(w(z))$. This subordination can be expressed in the form of $f(z) \prec g(z)$. 
Ma \& Minda (1994) combined diverse subclasses of convex and starlike functions. They cogitate an analytic function $\Phi$ with positive real part in $\Delta$, where $\Phi(0)=1$, $\Phi^{\prime}(0)>0$ that maps $\Delta$ onto a region starlike with respect to 1 and symmetric with respect to the real axis. This series of expansion for function $\Phi$ can be demonstrated in the form of

$$
\Phi(z)=1+B_{1} z+B_{2} z^{2}+\cdots,\left(B_{1}>0\right)
$$

The classes of Ma-Mindastarlike and Ma-Minda convex functions consist of functions $f \in$ A satisfying the subordination

$$
\frac{z f^{\prime}(z)}{f(z)} \prec \Phi(z)
$$

and

$$
1+\frac{z f^{\prime \prime}(z)}{f^{\prime}(z)} \prec \Phi(z)
$$

respectively. Both $f$ and $f^{-1}$ must be Ma-Minda starlike or convex, autonomously in order for function $f$ to be bistarlike or bi-convex of Ma-Minda type. $S T_{S}(\Phi)$ denote the class for bi-starlike of Ma-Minda type and $C V_{\zeta}(\Phi)$ denote the class of bi-convex of Ma-Minda type.

In modern works, several authors such as Lashin (2016), Crisan (2013) and Ali et al.(2012) introduced new subclasses of class sand obtained the estimates of the initial coefficients of the Taylor-Maclaurin for functions belonging to these classes.

In this paper, approximations of the initial coefficients for bi-univalent of Ma-Mindastarlike and convex functions were studied. Further, the upper bound of the Fekete-Szegö functional also studied.

\section{METHODS}

Definition 1. A function $f(z) \in \varsigma$ given by (1) is said to be in a class $\mathcal{K}_{\zeta}(\Phi, \alpha)$ with $0<\alpha \leq 1$ if the following conditions are satisfied:

$\frac{2\left(z f^{\prime}(z)+\alpha z^{2} f^{\prime \prime}(z)\right)}{(1-\alpha)(f(z)-f(-z))+\alpha z(f(z)-f(-z))^{\prime}} \prec \Phi(z)$ and

$$
\begin{gathered}
\frac{2\left(w g^{\prime}(w)+\alpha w^{2} g^{\prime \prime}(w)\right)}{(1-\alpha)(g(w)-g(-w))+\alpha w(g(w)-g(-w))^{\prime}} \\
<\Phi(w)
\end{gathered}
$$

and the function $g=f^{-1}(w)$ is given by (4).

We first declare the well-known lemmas that will be used to acquire the upper bounds of the initial coefficients and the Fekete-Szegö functional for function $f \in \mathcal{K}_{\zeta}(\Phi, \alpha)$.

Lemma 2.(Duren, 1983)If $p \in P$ then $\left|p_{k}\right| \leq 2$ for each $k$, where $P$ is the family of all functions $p$ analytic in $\Delta$, $\operatorname{Re}(p(z))>0$

$$
p(z)=1+p_{1} z+p_{2} z^{2}+p_{3} z^{3}+\cdots
$$

for $z \in \Delta$.

Lemma 3. (Zaprawa, 2014) Let $k, l \in \mathbb{R}$ and $z_{1}, z_{2} \in \mathbb{C}$. If $\left|z_{1}\right|<R$ and $\left|z_{2}\right|<R$ then,

$$
\left|(k+l) z_{1}+(k-l) z_{2}\right| \leq \begin{cases}2|k| R & \text { for }|k| \geq|l| \\ 2|l| R & \text { for }|k| \leq|l|\end{cases}
$$

\section{RESULTS}

For functions $\mathcal{K}_{\zeta}(\Phi, \alpha)$, the following coefficient estimates are obtained as given by Theorem 4 .

Theorem 4. Let $f$ given by (1) be in the class $\mathcal{K}_{\varsigma}(\Phi, \alpha)$ where $0<\alpha \leq 1$. Then

$$
\left|a_{2}\right| \leq \frac{B_{1} \sqrt{B_{1}}}{\sqrt{2\left|B_{1}^{2}(1+2 \alpha)+2(1+\alpha)^{2}\left(B_{1}-B_{2}\right)\right|}}
$$

and

$$
\left|a_{3}\right| \leq \frac{1}{2} B_{1}\left(\frac{1}{(1+2 \alpha)}+\frac{1}{2(1+\alpha)^{2}} B_{1}\right)
$$

Proof. For $f \in \mathcal{K}_{\varsigma}(\Phi, \alpha), g=f^{-1}$, there exist analytic functions $u, v: D \rightarrow D$ with $u(0)=v(0)=0$, satisfying

$$
\begin{gathered}
\frac{2\left(z f^{\prime}(z)+\alpha z^{2} f^{\prime \prime}(z)\right)}{(1-\alpha)(f(z)-f(-z))+\alpha z(f(z)-f(-z))^{\prime}} \\
=\Phi[u(z)]
\end{gathered}
$$

and

$$
\begin{gathered}
\frac{2\left(w g^{\prime}(w)+\alpha w^{2} g^{\prime \prime}(w)\right)}{(1-\alpha)(g(w)-g(-w))+\alpha w(g(w)-g(-w))^{\prime}} \\
=\Phi[v(w)]
\end{gathered}
$$


The functions $b$ and $c$ are defined as:

$$
b(z):=\frac{1+u(z)}{1-u(z)}=1+b_{1} z+b_{2} z^{2}+\cdots
$$

and

$$
c(z):=\frac{1+v(z)}{1-v(z)}=1+c_{1} z+c_{2} z^{2}+\cdots
$$

or it is equivalent to

$$
u(z)=\frac{1}{2}\left[b_{1} z+\left(b_{2}-\frac{c_{1}^{2}}{2}\right) z^{2}+\cdots\right]
$$

and

$$
v(z)=\frac{1}{2}\left[c_{1} z+\left(c_{2}-\frac{c_{1}^{2}}{2}\right) z^{2}+\cdots\right]
$$

Functions $b$ and $c$ are analytic in $\Delta$ with $b(0)=1=c(0)$. Since $b, c: \Delta \rightarrow \Delta$, the functions $b$ and $c$ have positive real parts in $\Delta$ and $\left|b_{i}\right| \leq 2$ and $\left|c_{i}\right| \leq 2$ for $i=1,2$.

From (5) and (11)-(14), we obtain

$$
\begin{gathered}
\frac{2\left(z f^{\prime}(z)+\alpha z^{2} f^{\prime \prime}(z)\right)}{(1-\alpha)(f(z)-f(-z))+\alpha z(f(z)-f(-z))^{\prime}} \\
=1+\frac{1}{2} B_{1} b_{1} z+\left[\frac{1}{2} B_{1}\left(b_{2}-\frac{b_{1}^{2}}{2}\right)+\frac{1}{4} B_{2} b_{1}^{2}\right] z^{2}+\cdots
\end{gathered}
$$

and

$$
\begin{gathered}
\frac{2\left(w g^{\prime}(w)+\alpha w^{2} g^{\prime \prime}(w)\right)}{(1-\alpha)(g(w)-g(-w))+\alpha w(g(w)-g(-w))^{\prime}} \\
=1+\frac{1}{2} B_{1} c_{1} w+\left[\frac{1}{2} B_{1}\left(c_{2}-\frac{c_{1}^{2}}{2}\right)+\frac{1}{4} B_{2} c_{1}^{2}\right] w^{2}+\cdots
\end{gathered}
$$

(17) and (18) will gives us

$$
\begin{gathered}
2(1+\alpha) a_{2}=\frac{1}{2} B_{1} b_{1} \\
2(1+2 \alpha) a_{3}=\frac{1}{2} B_{1} b_{2}-\frac{1}{4} B_{1} b_{1}^{2}+\frac{1}{4} B_{2} b_{1}^{2} \\
-2(1+\alpha) a_{2}=\frac{1}{2} B_{1} c_{1}
\end{gathered}
$$

and

$$
2(1+2 \alpha)\left(2 a_{2}^{2}-a_{3}\right)=\frac{1}{2} B_{1} c_{2}-\frac{1}{4} B_{1} c_{1}^{2}+\frac{1}{4} B_{2} c_{1}^{2}
$$

From (19) and (21), we get

$$
b_{1}=-c_{1}
$$

$$
8(1+\alpha)^{2} a_{2}^{2}=\frac{1}{4} B_{1}^{2}\left(b_{1}^{2}+c_{1}^{2}\right)
$$

By considering (20), (22) and (24), we obtain

$$
a_{2}^{2}=\frac{B_{1}^{3}\left(b_{2}+c_{2}\right)}{8 B_{1}^{2}(1+2 \alpha)+16(1+\alpha)^{2}\left(B_{1}-B_{2}\right)}
$$

By applying triangle inequality and Lemma 2 for the coefficients $b_{2}$ and $c_{2}$ into equation (25), we finally get:

$$
\left|a_{2}\right| \leq \frac{B_{1} \sqrt{B_{1}}}{\sqrt{2\left|B_{1}^{2}(1+2 \alpha)+2(1+\alpha)^{2}\left(B_{1}-B_{2}\right)\right|}}
$$

This gives the bound on $\left|a_{2}\right|$ as stated in (9).

Next, we apply (20) and (22) in order to find the bound on $\left|a_{3}\right|$, we will get

$$
a_{3}=a_{2}^{2}+\frac{B_{1}\left(b_{2}-c_{2}\right)}{8(1+2 \alpha)}
$$

By replacing $a_{2}^{2}$ from (25) into (26), we obtain

$$
a_{3}=\frac{B_{1}^{2}\left(b_{1}^{2}+c_{1}^{2}\right)}{32(1+\alpha)^{2}}+\frac{B_{1}\left(b_{2}-c_{2}\right)}{8(1+2 \alpha)}
$$

Lastly, by applying triangle inequality and Lemma 3 for the coefficients $b_{1}, b_{2}, c_{1}$ and $c_{2}$ in(27), we obtain:

$$
\left|a_{3}\right| \leq \frac{1}{2} B_{1}\left(\frac{1}{(1+2 \alpha)}+\frac{1}{2(1+\alpha)^{2}} B_{1}\right)
$$

Theorem 4 is completely proven.

The nexttheorem concerning the Fekete-Szegö inequality for $\mathcal{K}_{S}(\Phi, \alpha)$ will be formulated as shown in Theorem 5 .

Theorem 5. Let $f$ given by (1) be in the class $\mathcal{K}_{\varsigma}(\Phi, \alpha)$ and $\mu \in \mathbb{R}$, then

$$
\left|a_{3}-\mu a_{2}^{2}\right| \leq\left\{\begin{array}{c}
\frac{B_{1}}{2(1+2 \alpha)} \\
\text { for }|1-\mu| \leq \mathcal{T}(\alpha) \\
\frac{B_{1}^{3}|1-\mu|}{\left|2 B_{1}^{2}(1+2 \alpha)+4(1+\alpha)^{2}\left(B_{1}-B_{2}\right)\right|} \\
\text { for }|1-\mu| \geq \mathcal{T}(\alpha)
\end{array}\right.
$$

where

$$
\mathcal{T}(\alpha)=\frac{1}{(1+2 \alpha)} \cdot\left|(1+2 \alpha)+2(1+\alpha)^{2}\left(\frac{B_{1}-B_{2}}{B_{1}^{2}}\right)\right|
$$

and 
Proof. Let $f$ given by (1) be in the class $\mathcal{K}_{\varsigma}(\Phi, \alpha)$ and $\mu \epsilon$ $\mathbb{R}$.

By using the definition of Fekete-Szego, $a_{3}-\mu a_{2}^{2}$ and applying equation (26), we obtain

$$
a_{3}-\mu a_{2}^{2}=\left[a_{2}^{2}+\frac{B_{1}\left(b_{2}-c_{2}\right)}{8(1+2 \alpha)}\right]-\mu a_{2}^{2}
$$

It follows that,

$$
\begin{aligned}
a_{3}-\mu a_{2}^{2}=B_{1}\left(\left[h(\mu)+\frac{1}{8(1+2 \alpha)}\right] b_{2}\right. \\
\left.+\left[h(\mu)-\frac{1}{8(1+2 \alpha)}\right] c_{2}\right)
\end{aligned}
$$

where

$$
h(\mu)=\frac{B_{1}^{2}(1-\mu)}{8 B_{1}^{2}(1+2 \alpha)+16(1+\alpha)^{2}\left(B_{1}-B_{2}\right)}
$$

By applying Lemma 2 and Lemma 3 into equation (30), we can conclude that

$$
\begin{aligned}
& \left|a_{3}-\mu a_{2}^{2}\right| \\
& \leq B_{1}\left\{\begin{array}{c}
2|h(\mu)|(2) \quad \text { for } \quad|h(\mu)| \geq \frac{1}{8(1+2 \alpha)} \\
2\left|\frac{1}{8(1+2 \alpha)}\right|(2) \text { for } \quad|h(\mu)| \leq \frac{1}{8(1+2 \alpha)}
\end{array}\right. \\
= & \left\{\begin{array}{lll}
4 B_{1}|h(\mu)| & \text { for } \quad|h(\mu)| \geq \frac{1}{8(1+2 \alpha)} \\
\frac{B_{1}}{2(1+2 \alpha)} & \text { for } \quad|h(\mu)| \leq \frac{1}{8(1+2 \alpha)}
\end{array}\right.
\end{aligned}
$$

Finally, Theorem 5 is proven.

Taking $\mu=1$ in Theorem 5 , we obtain the following corollary.

Corollary 6. If $f(z)$ is given by equation (1) be in the class $\mathcal{K}_{\varsigma}(\Phi, \alpha)$, then

$$
\left|a_{3}-a_{2}^{2}\right| \leq \frac{B_{1}}{2(1+2 \alpha)}
$$

\section{SUMMARY}

Predominantly, the subclass of bi-univalent functions, $\mathcal{K}_{\varsigma}(\Phi, \alpha)$, is proposed using subordination. Then, the upper bound for the coefficients estimates and the FeketeSzegö functional are obtained for function in the class $\mathcal{K}_{\varsigma}(\Phi, \alpha)$. 


\section{REFERENCES}

Ali, R.M., Lee, S.K., Ravichandran, V. \& Supramaniam, S. Magesh, N. \& Yamini, J. (2013). Coefficient bounds for (2012). Coefficient estimates for bi-univalent Macertain subclasses of bi-univalent functions. Int. Mindastarlike and convex functions. Applied Mathematics Letters, 25, 344-351.

Math. Forum,8, 1337-1344.

Murugusundaramoorthy, G., Magesh, N. \& Prameela, V. (2013). Coefficient bounds for certain subclasses of bi-univalent function. Abstract and Applied Analysis, 3 pages.

NATO Advanced Study Institute held at the University of Durham, Durham, July 1-20, 1979, Academy Press, London, New York.

Brannan, D.A., Clunie, J.\& Kirwan, W.E. (1970). Coefficient estimates for a class of starlike functions. Canadian Mathematical Society, 22, 476-485.

Brannan, D.A.\& Taha, T.S. (1987). On some classes of biunivalent functions. Studia Universitatis Babes-Bolyai Mathematica, 31 (2), 70-77.

Bulut, S. (2013). Coefficient estimates for a class of analytic and bi-univalent functions. Novi Sad J. Math., 43 (2), $59-65$.

Crisan, O. (2013). Coefficient Estimates for Certain Subclasses of Bi-Univalent Functions. Gen. Math. Notes, 16, 93-102.

Das, R.N.\& Singh, P. (1977). On Subclass of Schlicht mapping. Indian Journal Pure Applied Mathematics, 8, 864-872.

Deniz, E. (2013). Certain subclasses of bi-univalent functions satisfying subordinate conditions. J. Class Anal., 2, 49-6o.

Duren, M.P. (1983). Univalent Functions, in Grundlehren Math. Wissenschaften, Springer-Verlag, Berlin, New York, Heidelberg and Tokyo.

Frasin, B.A. \& Aouf, M.K. (2011). New subclasses of biunivalent functions. Applied Mathematics Letter, 25, 1569-1573.

Lashin, A.Y. (2016). On certain subclasses of analytic and bi-univalent functions. Journal of the Egyptian Mathematical Society, 24, 220-225.

Lewin, M. (1967). On a coefficient problem for bi-univalent functions. Proceedings of the American Mathematical Society, 18, 63-68.

Ma, W.C. \& Minda, D. (1994). A unified treatment of some special classes of univalent functions. Proceedings of the Conference on Complex Analysis, Tianjin, 1992, Conf. Proc. Lecture Notes Anal., vol. I, pp. 157-169, International Press, Cambridge. 